\section{A RAINBOW OF INFECTION CONTROL WIPES}

Protection against the polio virus, MRSA and HIV is available by using Dürr Dental's FD312 surface disinfectant wipes. These handy cross-infection control wipes contain an aldehyde and phenol-free disinfectant with many applications including safe use on all washable surfaces and objects.

With no alcohol in its formulation, the integral disinfectant is ideal for use on dental chairs as it will not damage upholstery so enabling

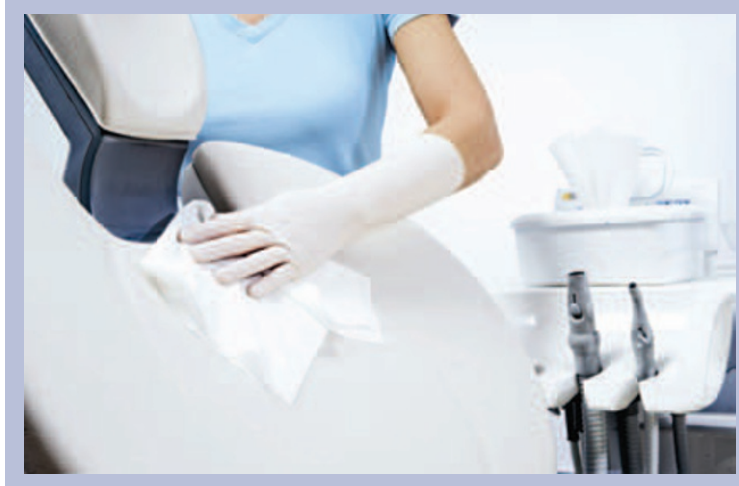

continuous wiping across chairs, tables and instrument trays. For floors too the wipes are easily adapted by using a retractable mop onto which they can be easily affixed for rapid cleaning. Effective against a wide spectrum of bacteria, fungi and viruses the disinfectant dries quickly and leaves no residue.

Dürr Dental manufacture a range of hygiene products of which FD312 wipes are just one. Conveniently colour coded to identify each product's application they range from blue for instruments, green for surfaces, pink for skin and hands, and yellow for special areas such as suction systems and amalgam separators.

\section{EFFECTIVE AND \\ CHLORHEXIDINE-FREE}

In light of recent enquiries, infection control expert schülke would like to confirm that none of its products sold in the UK contain the active ingredient chlorhexidine in the finished formulation, ensuring all products are safe to use.

The Medical Device Alert issued by the MHRA in October has caused many dental professionals to re-evaluate the products they use in the dental practice. With several reports reaching the MHRA of patients suffering dangerous anaphylactic reactions to the chemical chlorhexidine, a warning has been issued across the UK healthcare industry.

The full schülke product range includes wipes and liquids scientifically proven to be effective against a wide spectrum of infections and diseases. With the new schülke Plus Rewards Scheme, dental practices can also make significant savings on repeat purchases.

For more information, call 01142543500 or visit www.schulke.co.uk.
PRISTINE INSTRUMENTS AT THE PRESS OF A BUTTON

Plandent supply all of your favourite infection control consumable products, at very competitive prices. Effective sterilisation of hand instruments begins with the Hydrim washer/ disinfector from SciCan supplied by Plandent. This simpleto-operate unit automatically washes, rinses, disinfects and dries your dental instruments at the press of a button. To fully personalise your Hydrim there are a range of accessories to suit your individual practice needs.

Statim autoclaves are available in range of sizes depending on the capacity you're looking for. These reliable and effective autoclaves process your instruments quickly, reducing instrument investment and increasing efficiency in your practice. Both the Hydrim and Statim are supported by Plandent's accredited nationwide service network.

Plandent's team are fully trained on the latest guidelines in infection control and are kept fully up to date with any changes, enabling them to provide you with current information, guidance and advice. Call Plandent free on 0500500322 today.

\section{ADD A NEW STRING TO YOUR BOW IN 2013}

Practitioners who aspire to treat more challenging dental implant cases can further develop their skills on a series of courses in 2013. The Bath Spa Dentistry Advanced Implantology Programme is

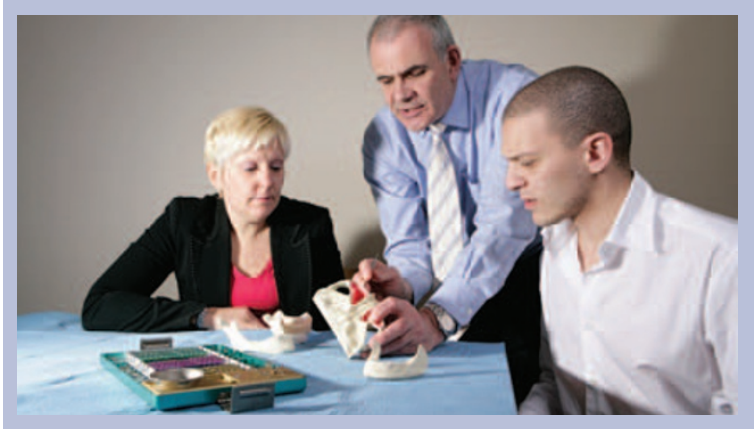

presented by Dermot McNulty. The four modules cover surgical skills, soft tissue management, bone grafting and sinus augmentation.

The programme is aimed at dentists with some experience of implantology who are ready to enhance their knowledge and skills. Delegates are taught via a combination of lectures, practical demonstrations and hands-on workshops, live surgical procedures, as well as patient management.

Dermot McNulty has extensive experience in implant and aesthetic dentistry and the treatment of complex restorative cases. He also runs an implantology year programme at Bath Spa Dentistry, which is now in its fifth year. Bath Spa Dentistry is a referral centre designed specifically for the delivery of advanced restorative and implant dentistry.

For booking and further information, call 01225443483 or email bathspa@ampmpa.co.uk

Further details can be found on the DENTSPLY website www. courses4implants.com. 